\title{
Optimal Algorithms for Right-Sizing Data Centers*
}

\author{
Susanne Albers \\ Technical University of Munich \\ albers@in.tum.de
}

\author{
Jens Quedenfeld \\ Technical University of Munich \\ jens.quedenfeld@in.tum.de
}

\begin{abstract}
Electricity cost is a dominant and rapidly growing expense in data centers. Unfortunately, much of the consumed energy is wasted because servers are idle for extended periods of time. We study a capacity management problem that dynamically right-sizes a data center, matching the number of active servers with the varying demand for computing capacity. We resort to a data-center optimization problem introduced by Lin, Wierman, Andrew and Thereska $[17,19]$ that, over a time horizon, minimizes a combined objective function consisting of operating cost, modeled by a sequence of convex functions, and server switching cost. All prior work addresses a continuous setting in which the number of active servers, at any time, may take a fractional value.

In this paper, we investigate for the first time the discrete datacenter optimization problem where the number of active servers, at any time, must be integer valued. Thereby we seek truly feasible solutions. First, we show that the offline problem can be solved in polynomial time. Our algorithm relies on a new, yet intuitive graph theoretic model of the optimization problem and performs binary search in a layered graph. Second, we study the online problem and extend the algorithm Lazy Capacity Provisioning (LCP) by Lin et al. $[17,19]$ to the discrete setting. We prove that LCP is 3-competitive. Moreover, we show that no deterministic online algorithm can achieve a competitive ratio smaller than 3. Hence, while LCP does not attain an optimal competitiveness in the continuous setting, it does so in the discrete problem examined here. We prove that the lower bound of 3 also holds in a problem variant with more restricted operating cost functions, introduced by Lin et al. [17].

Finally, we address the continuous setting and give a lower bound of 2 on the best competitiveness of online algorithms. This matches an upper bound by Bansal et al. [5]. A lower bound of 2 was also recently shown by Antoniadis and Schewior [3]. We develop an independent proof that extends to the scenario with more restricted operating cost.
\end{abstract}

\section{KEYWORDS}

Homogeneous servers; polynomial-time offline algorithm; online algorithm; lower bounds; discrete setting.

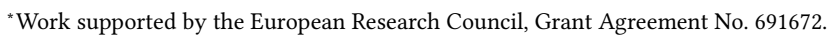

\section{(c) $(1) \Theta \Theta$}

This work is licensed under a Creative Commons Attribution-NonCommercial-NoDerivs International 4.0 License.

(C) 2018 Copyright held by the owner/author(s).

ACM ISBN 978-1-4503-5799-9/18/07.

https://doi.org/10.1145/3210377.3210385

\section{ACM Reference Format:}

Susanne Albers and Jens Quedenfeld. 2018. Optimal Algorithms for RightSizing Data Centers. In SPAA '18: SPAA '18: 30th ACM Symposium on Parallelism in Algorithms and Architectures, Fuly 16-18, 2018, Vienna, Austria. ACM, New York, NY, USA, 10 pages. https://doi.org/10.1145/3210377.3210385

\section{INTRODUCTION}

Energy conservation in data centers is a major concern for both operators and the environment. In the U.S., about $1.8 \%$ of the total electricity consumption is attributed to data centers [22]. In 2015, more than 416 TWh (terawatt hours) were used by the world's data centers, which exceeds the total power consumption in the UK [7]. Electricity cost is a significant expense in data centers [9]; about $18-28 \%$ of their budget is invested in power $[8,13]$. Remarkably, the servers of a data center are only utilized $20-40 \%$ of the time on average [4, 6]. Even worse, when idle and in active mode, they consume about half of their peak power [21]. Hence, a promising approach for energy conservation and capacity management is to transition idle servers into low-power sleep states. However, state transitions, and in particular power-up operations, also incur energy/cost. Therefore, dynamically matching the number of active servers with the varying demand for computing capacity is a challenging optimization problem. In essence, the goal is to right-size a data center over time so as to minimize energy and operation costs.

Problem Formulation. We investigate a basic algorithmic problem with the objective of dynamically resizing a data center. Specifically, we resort to a framework that was introduced by Lin, Wierman, Andrew and Thereska $[17,19]$ and further explored, for instance, in [1-3, 5, 18, 20, 23].

Consider a data center with $m$ homogeneous servers, each of which has an active state and a sleep state. An optimization is performed over a discrete, finite time horizon consisting of time steps $t=1, \ldots, T$. At any time $t, 1 \leq t \leq T$, a non-negative convex cost function $f_{t}(\cdot)$ models the operating cost of the data center. More precisely, $f_{t}\left(x_{t}\right)$ is the incurred cost if $x_{t}$ servers are in the active state at time $t$, where $0 \leq x_{t} \leq m$. This operating cost captures, e.g., energy cost and service delay, for an incoming workload, depending on the number of active servers. Furthermore, at any time $t$ there is a switching cost, taking into account that the data center may be resized by changing the number of active servers. This switching cost is equal to $\beta\left(x_{t}-x_{t-1}\right)^{+}$, where $\beta$ is a positive real constant and $(x)^{+}=\max (0, x)$. Here we assume that transition cost is incurred when servers are powered up from the sleep state to the active state. A cost of powering down servers may be folded into this cost. The constant $\beta$ incorporates, e.g., the energy needed to transition a server from the sleep state to the active state, as well as delays resulting from a migration of data and connections. We assume that at the beginning and at the end of the time horizon all servers are in the sleep state, i.e. $x_{0}=x_{T+1}=0$. The goal is to determine a vector $X=\left(x_{1}, \ldots, x_{T}\right)$ called schedule, 
specifying at any time the number of active servers, that minimizes

$$
\sum_{t=1}^{T} f_{t}\left(x_{t}\right)+\beta \sum_{t=1}^{T}\left(x_{t}-x_{t-1}\right)^{+} .
$$

In the offline version of this data-center optimization problem, the convex functions $f_{t}, 1 \leq t \leq T$, are known in advance. In the online version, the $f_{t}$ arrive over time. At time $t$, function $f_{t}$ is presented. Recall that the operating cost at time $t$ depends for instance on the incoming workload, which becomes known only at time $t$.

All previous work on the data-center optimization problem assumes that the server numbers $x_{t}, 1 \leq t \leq T$, may take fractional values. That is, $x_{t}$ may be an arbitrary real number in the range $[0, m]$. From a practical point of view this is acceptable because a data center has a large number of machines. Nonetheless, from an algorithmic and optimization perspective, the proposed algorithms do not compute feasible solutions. Important questions remain if the $x_{t}$ are indeed integer valued: (1) Can optimal solutions be computed in polynomial time? (2) What is the best competitive ratio achievable by online algorithms? In this paper, we present the first study of the data-center optimization problem assuming that the $x_{t}$ take integer values and, in particular, settle questions (1) and (2).

Previous Work. As indicated above, all prior work on the datacenter optimization problem assumes that the $x_{t}, 1 \leq t \leq T$, may take fractional values in $[0, m]$. First, Lin et al. [19] consider the offline problem. They develop an algorithm based on a convex program that computes optimal solutions. Second, Lin et al. [19] study the online problem. They devise a deterministic algorithm called Lazy Capacity Provisioning (LCP) and prove that it achieves a competitive ratio of exactly 3 . Algorithm LCP, at any time $t$, computes a lower bound and an upper bound on the number of active servers by considering two scenarios in which the switching cost $\beta$ is charged, either when a server is powered up or when it is powered down. The LCP algorithm lazily stays within these two bounds. The tight bound of 3 on the competitiveness of LCP also holds if the algorithm has a finite prediction window $w$, i.e. a time $t$ it knows the current as well as the next $w$ arriving functions $f_{t}, \ldots, f_{t+w}$. Furthermore, Lin et al. [19] perform an experimental study with two real-world traces evaluating the savings resulting from right-sizing in data centers.

Bansal et al. [5] presented a 2-competitive online algorithm and showed that no deterministic or randomized online strategy can attain a competitiveness smaller than 1.86. Recently, Antoniadis and Schewior [3] improved the lower bound to 2. Bansal et al. [5] also gave a 3-competitive memoryless algorithm and showed that this is the best competitive factor achievable by a deterministic memoryless algorithm. The data-center optimization problem is an online convex optimization problem with switching costs. Andrew et al. [1] showed that there is an algorithm with sublinear regret but that $O(1)$-competitiveness and sublinear regret cannot be achieved simultaneously. Antoniadis et al. [2] examine generalized online convex optimization, where the values $x_{t}$ selected by an algorithm may be points in a metric space, and relate it to convex body chasing.

Further work on energy conservation in data center includes, for instance, [14, 15]. Khuller et al. [14] introduce a machine activation problem. There exists an activation cost budget and jobs have to be scheduled on the selected, activated machines so as to minimize the makespan. They present algorithms that simultaneously approximate the budget and the makespan. A second paper by Li and Khuller [15] considers a generalization where the activation cost of a machine is a non-decreasing function of the load. In the more applied computer science literature, power management strategies and the value of sleep states have been studied extensively. The papers focus mostly on experimental evaluations. Articles that also present analytic results include [10-12].

Our Contribution. We conduct the first investigation of the discrete data-center optimization problem, where the values $x_{t}$, specifying the number of active servers at any time $t \in\{1, \ldots, T\}$, must be integer valued. Thereby, we seek truly feasible solutions.

First, in Section 2 we study the offline algorithm. We show that optimal solutions can be computed in polynomial time. Our algorithm is different from the convex optimization approach by Lin et al. [19]. We propose a new, yet natural graph-based representation of the discrete data-center optimization problem. We construct a grid-structured graph containing a vertex $v_{t, j}$, for each $t \in\{1, \ldots, T\}$ and $j \in\{0, \ldots, m\}$. Edges represent right-sizing operations, i.e. changes in the number of active servers, and are labeled with operating and switching costs. An optimal solution could be determined by a shortest path computation. However, the resulting algorithm would have a pseudo-polynomial running time. Instead, we devise an algorithm that improves solutions iteratively using binary search. In each iteration the algorithm uses only a constant number of graph layers. The resulting running time is $O(T \log m)$.

The remaining paper focuses on the online problem and develops tight bounds on the competitiveness. In Section 3 we adapt the LCP algorithm by Lin et al. [19] to the discrete data-center optimization problem. We prove that LCP is 3-competitive, as in the continuous setting. We remark that our analysis is different from that by Lin et al. [19]. Specifically, our analysis resorts to the discrete structure of the problem and identifies respective properties. The analysis by Lin et al. [19] relates to their convex optimization approach that characterizes optimal solutions in the continuous setting.

In Section 4 we devise lower bounds. We prove that no deterministic online algorithm can obtain a competitive ratio smaller than 3 . Hence, LCP achieves an optimal competitive factor. Interestingly, while LCP does not attain an optimal competitiveness in the continuous data-center optimization problem (where the $x_{t}$ may take fractional values), it does so in the discrete problem. We prove that the lower bound of 3 on the best possible competitive ratio also holds for a more restricted setting, originally introduced by Lin et al. [17] in the conference publication of their paper. Specifically, the problem is to find a vector $X=\left(x_{1}, \ldots, x_{T}\right)$ that minimizes

$$
\sum_{t=1}^{T} x_{t} f\left(\lambda_{t} / x_{t}\right)+\beta \sum_{t=1}^{T}\left(x_{t}-x_{t-1}\right)^{+}
$$

subject to $x_{t} \geq \lambda_{t}$, for $t \in\{1, \ldots, T\}$. Here $\lambda_{t}$ is the incoming workload at time $t$ and $f(z)$ is a non-negative convex function representing the operating cost of a single server running with load $z \in[0,1]$. Since $f$ is convex, it is optimal to distribute the jobs equally to all active servers, so that the operating cost at time $t$ is $x_{t} f\left(\lambda_{t} / x_{t}\right)$. This problem setting is more restricted in that there is only a single function $f$ modeling operating cost over the 


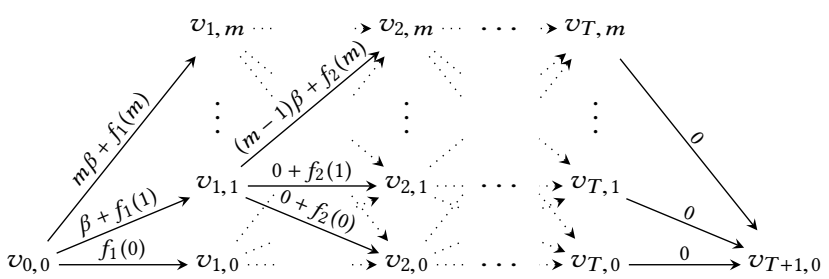

Figure 1: Construction of the graph.

time horizon. Nonetheless it is well motivated by real data center environments.

Furthermore, in Section 4 we address the continuous data-center optimization problem and prove that no deterministic online algorithm can achieve a competitive ratio smaller than 2 . The same result was shown by Antoniadis and Schewior [3]. We develop an independent proof that can again be extended to the more restricted optimization problem stated in (2), i.e. the lower bound of 2 on the best competitiveness holds in this setting as well.

Finally, in Section 4 we analyze online algorithms with a finite prediction window, i.e. at time $t$ an online algorithm knows the current as well as the next $w$ arriving functions $f_{t}, \ldots, f_{t+w}$. We show that all our lower bounds, for both settings (continuous and discrete) and both models (general and restricted), still hold.

\section{AN OPTIMAL OFFLINE ALGORITHM}

In this section we study the offline version of the discrete datacenter optimization problem. We develop an algorithm that computes optimal solutions in $O(T \log m)$ time.

\subsection{Graph-based approach}

Our algorithm works with an underlying directed, weighted graph $G=(V, E)$ that we describe first. Let $[k]:=\{1,2, \ldots, k\}$ and $[k]_{0}:=$ $\{0,1, \ldots, k\}$ with $k \in \mathbb{N}$. For each $t \in[T]$ and each $j \in[m]_{0}$, there is a vertex $v_{t, j}$, representing the state that exactly $j$ servers are active at time $t$. Furthermore, there are two vertices $v_{0,0}$ and $v_{T+1,0}$ for the initial and final states $x_{0}=0$ and $x_{T+1}=0$. For each $t \in\{2, \ldots, T\}$ and each pair $j, j^{\prime} \in[m]_{0}$, there is a directed edge from $v_{t-1, j}$ to $v_{t, j^{\prime}}$ having weight $\beta\left(j^{\prime}-j\right)^{+}+f_{t}\left(j^{\prime}\right)$. This edge weight corresponds to the switching cost when changing the number of servers between time $t-1$ and $t$ and to the operating cost incurred at time $t$. Similarly, for $t=1$ and each $j^{\prime} \in[m]_{0}$, there is a directed edge from $v_{0,0}$ to $v_{1, j^{\prime}}$ with weight $f_{1}\left(j^{\prime}\right)+\beta\left(j^{\prime}\right)^{+}$. Finally, for $t=T$ and each $j \in[m]_{0}$, there is a directed edge from $v_{T, j}$ to $v_{T+1,0}$ of weight 0 . The structure of $G$ is depicted in Figure 1.

In the following, for each $j \in[m]_{0}$, vertex set $R_{j}=\left\{v_{t, j} \mid t \in\right.$ $[T]\}$ is called row $j$. For each $t \in[T]$, vertex set $C_{t}=\left\{v_{t, j} \mid j \in\right.$ $\left.[\mathrm{m}]_{0}\right\}$ is called column $t$.

A path between $v_{0}$ and $v_{T+1}$ represents a schedule. If the path visits $v_{t, j}$, then $x_{t}=j$ servers are active at time $t$. Note that a path visits exactly one vertex in each column $C_{t}, 1 \leq t \leq T$, because the directed edges connect adjacent columns. The total length (weight) of a path is equal to the cost of the corresponding schedule. An optimal schedule can be determined using a shortest path computation, which takes $O(T m)$ time in the particular graph $G$. However, this running time is not polynomial because the encoding length of an input instance is linear in $T$ and $\log m$, in addition to the encoding of the functions $f_{t}$.

In the following, we present a polynomial time algorithm that improves an initial schedule iteratively using binary search. In each iteration the algorithm constructs and uses only a constant number of rows of $G$.

\subsection{Polynomial time algorithm}

An instance of the data-center optimization problem is defined by the tuple $\mathcal{P}=(T, m, \beta, F)$ with $F=\left(f_{1}, \ldots, f_{T}\right)$. We assume that $m$ is a power of two. If this is not the case we can transform the given problem instance $\mathcal{P}=(T, m, \beta, F)$ to $\mathcal{P}^{\prime}=\left(T, m^{\prime}, \beta, F^{\prime}\right)$ with $m^{\prime}=2^{\lceil\log m\rceil}$ and

$$
f_{t}^{\prime}(x)= \begin{cases}f_{t}(x) & x \leq m \\ x \cdot\left(f_{t}(m)+\epsilon\right) & \text { otherwise }\end{cases}
$$

with $\epsilon>0$. The term $x \cdot f_{t}(m)$ ensures that $f_{t}^{\prime}(x)$ is a convex function, since the greatest slope of $f_{t}$ is $f_{t}(m)-f_{t}(m-1) \leq f_{t}(m)$. The inequality holds because $f_{t}(x) \geq 0$ for all $x \in[m]_{0}$. The additional term $x \cdot \epsilon$ ensures that it is adverse to use a state $x>m$, because the cost of $f_{t}(m)$ is always smaller.

Our algorithm uses $\log m-1$ iterations denoted reversely by $k=K:=\log m-2$ for the first iteration and $k=0$ for the last iteration. The states used in iteration $k$ are always multiples of $2^{k}$. For the first iteration we use the rows $R_{0}, R_{m / 4}, R_{m / 2}, R_{3 m / 4}, R_{m}$, so that the graph of the first iteration contains the vertices

$$
V^{K}:=\left\{v_{0,0}, v_{T+1,0}\right\} \cup\left\{v_{t, \xi \cdot m / 4} \mid t \in[T], \xi \in\{0,1,2,3,4\}\right\} .
$$

The optimal schedule for this simplified problem instance can be calculated in $O(T)$ time, since each column contains only five states. Given an optimal schedule $\hat{X}^{k}=\left(\hat{x}_{1}^{k}, \ldots, \hat{x}_{T}^{k}\right)$ of iteration $k$, let

$$
V_{t}^{k-1}:=\left\{\hat{x}_{t}^{k}+\xi \cdot 2^{k-1} \mid \xi \in\{-2,-1,0,1,2\}\right\} \cap[m]_{0}
$$

be the states used in the $t$-th column of the next iteration $k-1$. Thus the iteration $k-1$ uses the vertex set

$$
V^{k-1}:=\left\{v_{0,0}, v_{T+1,0}\right\} \cup\left\{v_{t, j} \mid t \in[T], j \in V_{t}^{k-1}\right\} .
$$

Note that the states with $\xi \in\{-2,0,2\}$ were already used in iteration $k$ and we just insert the intermediate states $\xi=-1$ and $\xi=1$. If $\hat{x}_{t}^{k}=0$ (or $\hat{x}_{t}^{k}=m$ ), then $\xi \in\{-2,-1\}$ (or $\xi \in\{1,2\}$ ) leads to negative states (or to states larger than $m$ ), thus the set $V_{t}^{k-1}$ is cut with $[m]_{0}$ to ensure that we only use valid states.

The last iteration $(k=0)$ provides an optimal schedule for the original problem instance as shown in the next section. The runtime of the algorithm is $O(T \cdot \log m)$ and thus polynomial.

\subsection{Correctness}

To prove the correctness of the algorithm described in the previous section we have to introduce some definitions:

Given the original problem instance $\mathcal{P}=(T, m, \beta, F)$, we define $\mathcal{P}_{k}$ (with $k \in[K]_{0}:=[\log m-2]_{0}$ ) as the data-center optimization problem where we are only allowed to use the states that are multiples of $2^{k}$. Let $M_{k}:=\left\{n \in[m]_{0} \mid n \bmod 2^{k}=0\right\}$, so $X$ is a feasible schedule for $\mathcal{P}_{k}$ if $x_{t} \in M_{k}$ holds for all $t \in[T]$. To express $\mathcal{P}_{k}$ as a tuple, we need another tuple element called $M$ which 
describes the allowed states, i.e. $x_{t} \in M$ for all $t \in[T]$. The original problem instance can be written as $\mathcal{P}=\left(T, m, \beta, F,[m]_{0}\right)$ and $\mathcal{P}_{k}=\left(T, m, \beta, F, M_{k}\right)$. Note that $\mathcal{P}_{0}=\mathcal{P}$. Let $\hat{X}^{k}=\left(\hat{x}_{1}^{k}, \ldots, \hat{x}_{T}^{k}\right)$ denote an optimal schedule for $\mathcal{P}_{k}$. In general, for any given problem instance $Q=(T, m, \beta, F, M)$, let $\Phi_{k}(Q):=\left(T, m, \beta, F, M \cap\left\{i \cdot 2^{k} \mid\right.\right.$ $i \in \mathbb{N}\})$, so $\Phi_{k}(\mathcal{P})=\mathcal{P}_{k}$.

Instead of using only states that are multiple of $2^{k}$ we can also scale a given problem instance $Q=(T, m, \beta, F, M)$ as follows. Let

$$
\Psi_{l}(Q):=\left(T, m / 2^{l}, \beta \cdot 2^{l}, F^{\prime}, M^{\prime}\right)
$$

with $M^{\prime}:=\left\{x / 2^{l} \mid x \in M\right\}, F^{\prime}=\left(f_{1}^{\prime}, \ldots, f_{T}^{\prime}\right)$ and $f^{\prime}(x)_{t}:=$ $f_{t}\left(x \cdot 2^{l}\right)$. Given a schedule $X=\left(x_{1}, \ldots, x_{T}\right)$ for $Q$ with $\operatorname{cost} C^{Q}(X)$, the corresponding schedule $X^{\prime}=\left(x_{1} / 2^{l}, \ldots, x_{T} / 2^{l}\right)$ for $\Psi_{l}(Q)$ has exactly the same cost, i.e. $C^{Q}(X)=C^{\Psi_{l}(Q)}\left(X^{\prime}\right)$. Note that the problem instance $\Psi_{k}\left(\mathcal{P}_{k}\right)$ uses all integral states less than or equal to $m / 2^{l}$, so there are no gaps.

Furthermore, we introduce a continuous version of any given problem instance $Q$ where fractional schedules are allowed. Let $\bar{Q}=(T, m, \beta, \bar{F},[0, m])$ with $\bar{F}=\left(\bar{f}_{1}, \ldots, \bar{f}_{T}\right)$ be the continuous extension of the problem instance $Q=(T, m, \beta, F, M)$, where $x_{t} \in$ $[0, m], \bar{f}_{t}:[0, m] \rightarrow \mathbb{R}_{\geq 0}$ and

$$
\bar{f}_{t}(x):= \begin{cases}f_{t}(x) & \text { if } x \in M \\ (\lceil x\rceil-x) f_{t}(\lfloor x\rfloor)+(x-\lfloor x\rfloor) f_{t}(\lceil x\rceil) & \text { else. }\end{cases}
$$

The operating cost of the fractional states is linearly interpolated, thus $\bar{f}_{t}$ is convex for all $t \in[T]$. Let $X^{*}=\left(x_{1}^{*}, \ldots, x_{T}^{*}\right) \in[0, m]^{T}$ be an optimal schedule for $\overline{\mathcal{P}}$.

The set of all optimal schedules for a given problem instance $Q$ is denoted by $\Omega(Q)$. Let $C_{[a, b]}^{Q}(X):=\sum_{t=a}^{b} f_{t}\left(x_{t}\right)+\sum_{t=a+1}^{b} \beta\left(x_{t}-\right.$ $\left.x_{t-1}\right)^{+}$be the cost during the time interval $\{a, a+1, \ldots, b\}$. We define $f_{0}(x):=0$, so $C_{[0, T]}^{Q}(X)=C^{Q}(X)$.

Now, we are able to prove the correctness of our algorithm. We begin with a simple lemma showing the relationship between the functions $\Phi$ and $\Psi$.

LEMma 2.1. The problem instances $\Phi_{k-l}\left(\Psi_{l}\left(\mathcal{P}_{l}\right)\right)$ and $\Psi_{l}\left(\mathcal{P}_{k}\right)$ are equivalent.

The lemma can be proven by simple calculations using the definitions of $\Phi$ and $\Psi$ as shown in the full version of this paper. The next technical lemma will be needed later. Informally, it demonstrates that optimal solutions of the reduced discrete problem and the above continuous problem behave similarly.

Lemma 2.2. Let $Y \in \Omega\left(\mathcal{P}_{k}\right)$ be an optimal schedule for $\mathcal{P}_{k}$ with $k \in[K]_{0}$. There exists an optimal solution $X^{*} \in \Omega(\overline{\mathcal{P}})$ such that

$$
\left(y_{t}-y_{t-1}\right) \cdot\left(x_{t}^{*}-x_{t-1}^{*}\right) \geq 0
$$

holds for all $t \in[T]$ with $\left|y_{t}-x_{t}^{*}\right| \geq 2^{k}$ or $\left|y_{t-1}-x_{t-1}^{*}\right| \geq 2^{k}$.

The proof (see full paper) consists of a careful case distinction according to the relations of $y_{t-1}, y_{t}, x_{t-1}$ and $x_{t}$. By using this lemma, we can show that an optimal solution for a discrete problem instance $\mathcal{P}_{k}$ cannot be very far from an optimal solution of the continuous problem instance $\overline{\mathcal{P}}$.
Lemma 2.3. Let $\hat{X}^{k} \in \Omega\left(\mathcal{P}_{k}\right)$ be an arbitrary optimal schedule for $\mathcal{P}_{k}$ with $k \in[K]_{0}$. There exists an optimal schedule $X^{*} \in \Omega(\overline{\mathcal{P}})$ for $\overline{\mathcal{P}}_{\text {such that }}\left|\hat{x}_{t}^{k}-x_{t}^{*}\right|<2^{k}$ holds for all $t \in[T]$. Formally,

$\forall k \in[K]_{0}: \forall \hat{X}^{k} \in \Omega\left(\mathcal{P}_{k}\right): \exists X^{*} \in \Omega(\overline{\mathcal{P}}): \forall t \in[T]:\left|\hat{x}_{t}^{k}-x_{t}^{*}\right|<2^{k}$.

Proof. To get a contradiction, we assume that there exists a $\hat{X}^{k} \in \Omega\left(\mathcal{P}_{k}\right)$ with $k \in[K]_{0}$ such that for all optimal schedules $X^{*} \in \Omega(\overline{\mathcal{P}})$ there is at least one $t \in[T]$ with $\left|\hat{x}_{t}^{k}-x_{t}^{*}\right| \geq 2^{k}$. Let $X^{*} \in \Omega(\overline{\mathcal{P}})$ be arbitrary. Given the schedule $\hat{X}^{k}$, let $J_{1}, \ldots, J_{l} \subseteq[T]$ be the inclusion maximal time intervals such that $\left|\hat{x}_{t}^{k}-x_{t}^{*}\right| \geq 2^{k}$ holds for all $t \in J_{j}$ and the sign of $\hat{x}_{t}^{k}-x_{t}^{*}$ remains the same during $J_{j}$. The set of all $J_{j}$ with $j \in[l]$ is denoted by $\mathcal{J}$. If $\mathcal{J}$ is empty, then the condition $\left|\hat{x}_{t}^{k}-x_{t}^{*}\right|<2^{k}$ is fulfilled for all $t \in[T]$. We divide $\mathcal{J}$ into the disjunct sets $\mathcal{J}^{+}$and $\mathcal{J}^{-}$such that $\mathcal{J}^{+}$contains the intervals where $\hat{x}_{t}^{k}-x_{t}^{*}$ is positive and $\mathcal{J}^{-}$the others.

Given a schedule $X$, the corresponding interval set is denoted by $\mathcal{J}(X)$, the set of all time slots by $S(X):=\{t \in J \mid J \in \mathcal{J}(X)\}$, and the number of time slots in $\mathcal{J}$ by $L(X):=|S(\mathcal{J}(X))|=\sum_{J \in \mathcal{J}}|J|$.

We will use a recursive transformation $\phi$ that reduces $L(X)$ at least by one for each step, while the cost of $X$ is not increased. Formally, we have to show that $L(\phi(X)) \leq L(X)-1$ and $C^{\overline{\mathcal{P}}}(\phi(X)) \leq$ $C^{\overline{\mathcal{P}}}(X)$ holds. The first inequality ensures that the recursive procedure will terminate. The transformation described below will produce fractional schedules, however for each $t \in[T] \backslash S(X)$ it is ensured that $x_{t} \in M_{k}$. Therefore, if $L(X)=0$, the corresponding schedule fulfills $\left|x_{t}-x_{t}^{*}\right|<2^{k}$ and $x_{t} \in M_{k}$ for all $t \in[T]$.

To describe the transformation, we will use the following notation: A given schedule $Y=\left(y_{1}, \ldots, y_{T}\right)$ with $L(Y)>0$ is transformed to $Z=\phi(Y)=\left(z_{1}, \ldots, z_{T}\right)$.

Let $J:=\left(t_{i}+1, \ldots, t_{i+1}-1\right) \in \mathcal{J}(Y)$. We differ between two cases, in case 1 we handle the intervals in $\mathcal{J}^{+}$, i.e. $y_{t}>x_{t}^{*}+2^{k}$ holds for all $t \in J$ and in case 2 we handle the intervals in $\mathcal{J}^{-}$, i.e. $y_{t}<x_{t}^{*}-2^{k}$. We will handle case 1 first.

Let $\lceil x\rceil_{n}:=n \cdot\lceil x / n\rceil$ with $x \in \mathbb{R}$ and $n \in \mathbb{N}$ be the smallest value that is divisible by $n$ and greater than or equal to $x$. The schedule $Y$ is transformed to $Z$ with

$$
z_{t}:= \begin{cases}y_{t} & \text { if } t \notin J \\ \lambda \cdot y_{t}+(1-\lambda) \cdot x_{t}^{*} & \text { if } t \in J\end{cases}
$$

where $\lambda \in[0,1]$ is as small as possible such that $z_{t} \geq\left\lceil x_{t}^{*}\right\rceil_{2^{k}}$ holds for all $t \in J$, so at least one time slot $t_{=} \in J$ satisfies this condition with equality. This transformation ensures that $L(Z) \leq L(Y)-1$ holds, because the interval $J$ is split into at least two intervals and one time slot $\left(t_{=}\right)$between them that fulfills $\left|z_{t_{=}}-x_{t_{=}}^{*}\right|<2^{k}$.

We still have to show that the total cost is not increased by this operation. The total cost can be written as

$$
\begin{aligned}
C^{\overline{\mathcal{P}}}(X)= & C_{\left[0, t_{i}\right]}^{\overline{\mathcal{P}}}(X)+\beta\left(x_{t_{i}+1}-x_{t_{i}}\right)^{+}+C_{\left[t_{i}+1, t_{i+1}-1\right]}^{\overline{\mathcal{P}}}(X) \\
& +\beta\left(x_{t_{i+1}}-x_{t_{i+1}-1}\right)^{+}+C_{\left[t_{i+1}, T\right]}^{\overline{\mathcal{P}}}(X) .
\end{aligned}
$$

We have $C_{\left[0, t_{i}\right]}^{\overline{\mathcal{P}}}(Y)=C_{\left[0, t_{i}\right]}^{\overline{\mathcal{P}}}(Z)$ and $C_{\left[t_{i+1}, T\right]}^{\overline{\mathcal{P}}}(Y)=C_{\left[t_{i+1}, T\right]}^{\overline{\mathcal{P}}}(Z)$.

Consider the time slot $t_{i}$. By the definition of the interval $J$, the condition $\left|y_{t_{i}+1}-x_{t_{i}+1}^{*}\right| \geq 2^{k}$ is fulfilled. Thus we can apply Lemma 2.2 which says that the terms $\left(y_{t_{i}+1}-y_{t_{i}}\right)$ and $\left(x_{t_{i}+1}^{*}-x_{t_{i}}^{*}\right)$ are both either non-negative or non-positive, so in Equation (4) the 
term $\beta\left(x_{t_{i}+1}-x_{t_{i}}\right)^{+}$can be replaced by $\beta\left(x_{t_{i}+1}-x_{t_{i}}\right)$ or zero, respectively. Analogously, for the time slot $t_{i+1}$, the condition $\left|y_{t_{i+1}-1}-x_{t_{i+1}-1}^{*}\right| \geq 2^{k}$ is fulfilled, so by Lemma 2.2 the term $\beta\left(x_{t_{i+1}}-x_{t_{i+1}-1}\right)^{+}$in Equation (4) can be replaced by $\beta\left(x_{t_{i+1}}-\right.$ $\left.x_{t_{i+1}-1}\right)$ or zero. In the former cases, the cost function is

$$
\begin{aligned}
C^{\overline{\mathcal{P}}}(X)= & C_{\left[0, t_{i}\right]}^{\overline{\mathcal{P}}}(X)+\beta x_{t_{i}+1}-\beta x_{t_{i}}+C_{\left[t_{i}+1, t_{i+1}-1\right]}^{\overline{\mathcal{P}}}(X) \\
& +\beta x_{t_{i+1}}-\beta x_{t_{i+1}-1}+C_{\left[t_{i+1}, T\right]}^{\overline{\mathcal{P}}}(X) .
\end{aligned}
$$

Given a schedule $X=\left(x_{1}, \ldots, x_{t}\right)$, we define $X_{[a, b]}:=\left(x_{a}, \ldots, x_{b}\right)$ and $X_{J}=X_{\left[t_{i}+1, t_{i+1}-1\right]}$. Since there is no summand that contains both $x_{t_{i}}$ and $x_{t_{i}+1}$, the function

$$
\begin{aligned}
D_{X^{*}}\left(\left(x_{t_{i}+1}^{\prime}, \ldots, x_{t_{i+1}-1}^{\prime}\right)\right):= & C_{\left[0, t_{i}\right]}^{\overline{\mathcal{P}}}\left(X^{*}\right)-\beta x_{t_{i}}^{*} \\
& +\beta x_{t_{i}+1}^{\prime}+C_{\left[t_{i}+1, t_{i+1}-1\right]}^{\overline{\mathcal{P}}}\left(X^{\prime}\right)+\beta x_{t_{i+1}}^{\prime} \\
& -\beta x_{t_{i+1}-1}^{*}+C_{\left[t_{i+1}, T\right]}^{\overline{\mathcal{P}}}\left(X^{*}\right)
\end{aligned}
$$

with $x_{t_{i}+1}^{\prime} \geq x_{t_{i}+1}^{*}$ and $x_{t_{i+1}-1}^{\prime} \geq x_{t_{i+1}-1}^{*}$ is convex and has a minimum at $X_{J}^{\min }:=\left(x_{t_{i}+1}^{*}, \ldots, x_{t_{i+1}-1}^{*}\right)$.

Due to convexity, $D_{X^{*}}\left(Y_{J}\right) \geq D_{X^{*}}\left(Z_{J}\right) \geq D_{X^{*}}\left(X_{J}^{\min }\right)$, because $Z_{J}=\lambda Y_{J}+(1-\lambda) X_{J}^{\mathrm{min}}$. Therefore $C^{\overline{\mathcal{P}}}(Z) \leq C^{\overline{\mathcal{P}}}(Y)$ holds. If $\beta\left(x_{t_{i}+1}-x_{t_{i}}\right)^{+}=0$ or $\beta\left(x_{t_{i+1}}-x_{t_{i+1}-1}\right)^{+}=0$ we can use the same argument.

We still have to handle the second case, i.e. $y_{t}<x_{t}^{*}$. The proof works almost analogously, the difference is that we choose $\lambda$ as small as possible such that $z_{t} \leq\left\lfloor x_{t}^{*}\right\rfloor_{2^{k}}$ (where $\left.\lfloor x\rfloor_{n}:=n \cdot\lfloor x / n\rfloor\right)$. Then we have a time slot $t_{=}$with $z_{t_{=}}=\left\lfloor x_{t_{=}}^{*}\right\rfloor_{2^{k}}$, so $L(Z) \leq L(Y)-1$. The proof that shows $C^{\overline{\mathcal{P}}}(Z) \leq C^{\overline{\mathcal{P}}}(Y)$ holds for both cases.

We use the transformation $\phi$ until $L(Z)=0$. Then $\mathcal{J}(Z)$ is empty, so all states of $Z$ are multiples of $2^{k}$, i.e. $z_{t} \in M_{k}$ for all $t \in[T]$. Since $\hat{X}^{k}$ was defined to be optimal, $C^{\overline{\mathcal{P}}}\left(\hat{X}^{k}\right)=C^{\overline{\mathcal{P}}}(Z)$ holds. By our assumption, $Z \neq \hat{X}^{k}$ holds (because otherwise $\left|\hat{x}_{t}^{k}-x_{t}^{*}\right|<2^{k}$ would be fulfilled for all $t \in[T]$ ), so there was a transformation with $\lambda<1$. Thus we moved towards the optimal schedule, however by $C^{\overline{\mathcal{P}}}\left(\hat{X}^{k}\right)=C^{\overline{\mathcal{P}}}(Z)$, the cost does not change. As $D_{X^{*}}\left(X^{\prime}\right)$ is a convex function, $C^{\overline{\mathcal{P}}}\left(\hat{X}^{k}\right)=C^{\overline{\mathcal{P}}}(Z)$ implies that $C^{\overline{\mathcal{P}}}(Z)=C^{\overline{\mathcal{P}}}\left(X^{*}\right)$, because $X^{*}$ minimizes $C^{\overline{\mathcal{P}}}$. In this case $\hat{X}^{k}$ is also optimal for $\overline{\mathcal{P}}$, so the condition $\left|\hat{x}_{t}^{k}-x_{t}^{*}\right|<2^{k}$ is already fulfilled.

In all cases we get a contradiction, so our assumption was wrong and the lemma is proven.

The next lemma shows how an optimal fractional schedule can be rounded to an integral schedule such that it is still optimal.

Lemma 2.4. Let $X^{*} \in \Omega(\overline{\mathcal{P}})$. The schedules $\left\lfloor X^{*}\right\rfloor:=\left(\left\lfloor x_{1}^{*}\right\rfloor, \ldots\right.$, $\left.\left\lfloor x_{T}^{*}\right\rfloor\right)$ and $\left\lceil X^{*}\right\rceil:=\left(\left\lceil x_{1}^{*}\right\rceil, \ldots,\left\lceil x_{T}^{*}\right\rceil\right)$ are optimal too, i.e. $\left\lfloor X^{*}\right\rfloor,\left\lceil X^{*}\right\rceil$ $\in \Omega(\overline{\mathcal{P}})$.

Proof. Let $X^{*} \in \Omega(\overline{\mathcal{P}})$ be arbitrary. Let $\mathcal{I}\left(X^{*}\right)=\left\{I_{1}, \ldots, I_{l}\right\}$ be the set of time intervals such that for each $I_{i}:=\left\{a_{i}, a_{i}+1, \ldots, b_{i}\right\}$ with $i \in[l]$ the following conditions are fulfilled

(1) All states of $X^{*}$ have the same value during $I_{i}$, i.e. $x_{t}^{*}=v_{i}$ for all $t \in I_{i}$.

(2) The value is fractional, i.e. $v_{i} \notin \mathbb{N}$.

(3) Each $I_{i}$ is inclusion maximal, i.e. $x_{a_{i}-1}^{*} \neq v_{i}$ and $x_{b_{i}+1}^{*} \neq v_{i}$.
(4) The intervals are sorted, i.e. $b_{i}<a_{i+1}$ for all $i \in[l-1]$.

If $\mathcal{I}\left(X^{*}\right)=\emptyset$, then $X^{*}$ is an integral schedule, so $\left\lfloor X^{*}\right\rfloor=X^{*}=\left\lceil X^{*}\right\rceil$. Otherwise let $I_{i} \in \mathcal{I}\left(X^{*}\right)$ be an arbitrary interval. We will transform $X^{*}$ to $X^{\prime}$ by changing the states at $I_{i}$ such that $\left|\mathcal{I}\left(X^{\prime}\right)\right|<\left|\mathcal{I}\left(X^{*}\right)\right|$ and $\left\lfloor x_{t}^{*}\right\rfloor \leq x_{t}^{\prime} \leq\left\lceil x_{t}^{*}\right\rceil$ for all $t \in I_{i}$. Let $g(x):=\sum_{t=a_{i}}^{b_{i}} \bar{f}_{t}(x)$. Since each $\bar{f}_{t}(x)$ is linear for $x \in\left[\left\lfloor v_{i}\right\rfloor,\left\lceil v_{i}\right\rceil\right]$, the slope of $g(x)$ is constant for $x \in\left[\left\lfloor v_{i}\right\rfloor,\left\lceil v_{i}\right\rceil\right]$ and denoted by $g^{\prime}\left(v_{i}\right)$. According to $I_{i}$, we have to differ between different cases:

(1) $x_{a_{i}-1}^{*}<v_{i}<x_{b_{i}+1}^{*}$

Let $\tilde{x}_{a_{i}-1}^{*}:=\max \left\{x_{a_{i}-1}^{*},\left\lfloor v_{i}\right\rfloor\right\}$ and $\tilde{x}_{b_{i}+1}^{*}:=\min \left\{x_{b_{i}+1}^{*},\left\lceil v_{i}\right\rceil\right\}$. By using any schedule with $x_{a_{i}}^{\prime}=x_{a_{i}+1}^{\prime}=\cdots=x_{b_{i}}^{\prime} \in$ $\left[\tilde{x}_{a_{i}-1}^{*}, \tilde{x}_{b_{i}+1}^{*}\right]$ (and $x_{t}^{\prime}=x_{t}^{*}$ otherwise), the switching cost is unchanged. Since $I_{i}$ is inclusion maximal and $X^{*}$ is optimal, we can conclude that $g^{\prime}\left(v_{i}\right)=0$, so $C\left(X^{\prime}\right)=C\left(X^{*}\right)$. To show that $\left\lfloor X^{*}\right\rfloor$ is optimal, we set $x_{t}^{\prime}=\tilde{x}_{b_{i}+1}^{*}$ for all $t \in I_{i}$. To show that $\left\lceil X^{*}\right\rceil$ is optimal, we set $x_{t}^{\prime}=\tilde{x}_{a_{i}-1}^{*}$ for all $t \in I_{i}$.

(2) $x_{a_{i}-1}^{*}>v_{i}>x_{b_{i}+1}^{*}$ This case works analogously to the first case

(3) $x_{a_{i}-1}^{*}>v_{i}<x_{b_{i}+1}^{*}$ Let $v^{+}=\min \left\{x_{a_{i}-1}^{*}, x_{b_{i}+1}^{*},\left\lceil v_{i}\right\rceil\right\}$. Let $v_{i}^{\prime} \in\left[\left\lfloor v_{i}\right\rfloor, v^{+}\right]$. By using the schedule $x_{a_{i}}^{\prime}=x_{a_{i}+1}^{\prime}=\cdots=x_{b_{i}}^{\prime}=v_{i}^{\prime}$ for all $t \in I_{i}$, the switching cost is increased by $\beta\left(v_{i}-v_{i}^{\prime}\right)$, but the operating cost is reduced by $g^{\prime}\left(v_{i}\right) \cdot\left(v_{i}-v_{i}^{\prime}\right)$. Since $v_{i}$ is fractional, $v_{i} \notin\left\{x_{a_{i}-1}^{*}, x_{b_{i}+1}^{*}\right\}$. As $X^{*}$ is optimal, we can conclude that $g^{\prime}\left(v_{i}\right)=\beta$, so the total cost of $X^{\prime}$ does not change for $v_{i}^{\prime} \in\left[\left\lfloor v_{i}\right\rfloor, v^{+}\right]$. To show that $\left\lfloor X^{*}\right\rfloor$ is optimal, we set $x_{t}^{\prime}=\left\lfloor v_{i}\right\rfloor$ for all $t \in I_{i}$. To show that $\left\lceil X^{*}\right\rceil$ is optimal, we set $x_{t}^{\prime}=v^{+}$for all $t \in I_{i}$.

(4) $x_{a_{i}-1}^{*}<v_{i}>x_{b_{i}+1}^{*}$

This case works analogously to the third case, but $\lfloor x\rfloor$ and $\lceil x\rceil$ are swapped as well as min and max. Furthermore, $g^{\prime}\left(v_{i}\right)$ $=-\beta$ and we replace $\left(v_{i}-v_{i}^{\prime}\right)$ with $\left(v_{i}^{\prime}-v_{i}\right)$.

By using the transformation described above, we can reduce the number $|\mathcal{I}|$ of fractional intervals is at least reduced by 1 . By applying the transformation several times until $|\mathcal{I}|=0$, we receive $\left\lfloor X^{*}\right\rfloor$ or $\left\lceil X^{*}\right\rceil$. The total cost is not increased by the operations.

So far, we have shown in Lemma 2.3 that for each optimal solution of the discrete problem instance $\mathcal{P}_{k}$ there is an optimal solution of the continuous problem instance $\overline{\mathcal{P}}$ that is not far away. In the following lemma we expand this statement: Given an optimal solution for $\mathcal{P}_{k}$, there is not only a fractional solution for $\overline{\mathcal{P}}$ that is not far away, but also an optimal solution of the discrete problem instance $\mathcal{P}_{l}$ for the subsequent iterations $l<k$.

LEMmA 2.5. Let $k>l$ with $k, l \in[K]_{0}$. Let $\hat{X}^{k} \in \Omega\left(\mathcal{P}_{k}\right)$ be an arbitrary optimal schedule for $\mathcal{P}_{k}$ with $k \in[K]_{0}$. There exists an optimal schedule $\hat{X}^{l} \in \Omega\left(\mathcal{P}^{l}\right)$ for $\mathcal{P}^{l}$ such that $\left|\hat{x}_{t}^{k}-\hat{x}_{t}^{l}\right| \leq 2^{k}$ for all $t \in[T]$. Formally, $\forall k \in[K]_{0}: \forall l \in[k-1]: \forall \hat{X}^{k} \in \Omega\left(\mathcal{P}_{k}\right): \exists \hat{X}^{l} \in$ $\Omega\left(\mathcal{P}_{l}\right): \forall t \in[T]:\left|\hat{x}_{t}^{k}-\hat{x}_{t}^{l}\right| \leq 2^{k}$.

Proof. Consider the reduced problem instance $Q:=\Psi_{l}\left(\mathcal{P}_{l}\right)$ as well as the problem instance $Q_{k-l}:=\Phi_{k-l}\left(\Psi_{l}\left(\mathcal{P}_{l}\right)\right)$ which is equivalent to $\left.\Psi_{l}\left(\mathcal{P}_{k}\right)\right)$ due to Lemma 2.1. Let $\hat{X}_{Q}^{k-l}=\left(\hat{x}_{1}^{k} / 2^{l}, \ldots, \hat{x}_{T}^{k} / 2^{l}\right)$ be an optimal schedule for $Q_{k-l}$. We apply Lemma 2.3, but we 
use $\hat{X}_{Q}^{k-l}$ and $Q$ instead of $\hat{X}^{k}$ and $\mathcal{P}$. By Lemma 2.3, there exists an optimal fractional schedule $X_{Q}^{*}=\left(x_{1}^{*}, \ldots, x_{T}^{*}\right)$ for $\bar{Q}$ such that $\left|\hat{x}_{t}^{k} / 2^{l}-x_{t}^{*}\right| \leq 2^{k-l}$. By Lemma 2.4, $\left\lfloor X_{Q}^{*}\right\rfloor$ is also an optimal schedule for $\bar{Q}$ and therefore it is also optimal for $Q$. The inequality $\left|\hat{x}_{t}^{k} / 2^{l}-\left\lfloor x_{t}^{*}\right\rfloor\right| \leq 2^{k-l}$ still holds, because the terms $\hat{x}_{t}^{k} / 2^{l}$ and $2^{k-l}$ are integral and therefore adding a value less than 1 to the left side cannot invalidate the inequality. Let $\hat{X}^{l}:=\left(\left\lfloor x_{1}^{*}\right\rfloor \cdot 2^{l}, \ldots,\left\lfloor x_{T}^{*}\right\rfloor \cdot 2^{l}\right)$. As $\left\lfloor X_{Q}^{*}\right\rfloor$ is optimal for $Q, \hat{X}^{l}$ must be optimal for $\mathcal{P}_{l}$. Furthermore $\left\lfloor x_{t}^{*}\right\rfloor=\hat{x}_{t}^{l} / 2^{l}$ holds, so we can insert it into the above inequality and get $\left|\hat{x}_{t}^{k} / 2^{l}-\hat{x}_{t}^{l} / 2^{l}\right| \leq 2^{k-l}$ which is equivalent to $\left|\hat{x}_{t}^{k}-\hat{x}_{t}^{l}\right| \leq 2^{k}$.

Now, we have proven all parts to show the correctness of our polynomial-time algorithm:

Theorem 2.6. The algorithm described in Section 2.2 is correct.

Proof. We will show the correctness by induction. In the first iteration, the algorithm finds an optimal schedule for $\mathcal{P}_{K}$, because all states of $M_{K}$ are considered.

Given an optimal schedule $\hat{X}^{k}$, in the next iteration the algorithm only considers the states $x_{t}$ with $\left|\hat{x}_{t}^{k}-x_{t}\right| \leq 2^{k}$. By Lemma 2.5 , there exists an optimal schedule $\hat{X}^{l}$ with $l=k-1$ such that $\mid \hat{x}_{t}^{k}-$ $\hat{x}_{t}^{l} \mid \leq 2^{k}$ holds. Therefore the schedule found in iteration $k-1$ must be optimal for $\mathcal{P}_{k-1}$ (although some states are ignored by the algorithm). Thus, by induction, the algorithm will find an optimal schedule for $\mathcal{P}_{0}=\mathcal{P}$ in the last iteration.

\section{AN OPTIMAL ONLINE ALGORITHM}

Lin et. al. [17, 19] developed an algorithm called Lazy Capacity Provisioning (LCP) that achieves a competitive ratio of 3 for the continuous setting (i.e. $x_{t} \in \mathbb{R}$ ). In this section we adapt LCP to the discrete data-center optimization problem and prove the algorithm is 3-competitive for this problem as well.

The general approach of our proof is similar to the proof of the continuous setting in [17]. Some lemmas (e.g., Lemma 3.1 and 3.6) were adopted, however, their proofs are completely different. Lin et. al. use the properties of the convex program, especially duality and the complementary slackness conditions. This approach cannot be adapted to the discrete setting.

\subsection{Algorithm}

First, we will define lower and upper bounds for the optimal offline solution that can be calculated online. For a given time slot $\tau$ let $X_{\tau}^{L}:=\left(x_{\tau, 1}^{L}, \ldots, x_{\tau, \tau}^{L}\right)$ be the vector that minimizes

$$
C_{\tau}^{L}(X)=\sum_{t=1}^{\tau} f_{t}\left(x_{t}\right)+\beta \sum_{t=1}^{\tau}\left(x_{t}-x_{t-1}\right)^{+}
$$

with $X=\left(x_{1}, \ldots, x_{\tau}\right)$. This term describes the cost of a workload that ends at $\tau \leq T$. For $\tau=T$ this equation is equivalent to (1). Let $x_{\tau}^{L}:=x_{\tau, \tau}^{L}$ be the last state for this truncated workload. If there is more than one vector that minimizes (5), then $x_{\tau}^{L}$ is defined as the smallest possible value.

Similarly, let $X_{\tau}^{U}:=\left(x_{\tau, 1}^{U}, \ldots, x_{\tau, \tau}^{U}\right)$ be the vector that minimizes

$$
C_{\tau}^{U}(X)=\sum_{t=1}^{\tau} f_{t}\left(x_{t}\right)+\beta \sum_{t=1}^{\tau}\left(x_{t-1}-x_{t}\right)^{+} .
$$

The difference to the equation (5) is that we pay the switching cost for powering down. Powering up does not cost anything. The last state is denoted by $x_{\tau}^{U}:=x_{\tau, \tau}^{U}$. If there is more than one vector that minimizes (6), then $x_{\tau}^{U}$ is the largest possible value.

Define $[x]_{a}^{b}:=\max \{a, \min \{b, x\}\}$ as the projection of $x$ into the interval $[a, b]$. The LCP algorithm is defined as follows:

$$
x_{\tau}^{\mathrm{LCP}}:= \begin{cases}0, & \tau=0 \\ {\left[x_{\tau-1}^{\mathrm{LCP}}\right]_{x_{\tau}^{L}}^{x_{\tau}^{U}},} & \tau \geq 1\end{cases}
$$

Before we can prove that this algorithm is 3-competitive, we have to introduce some notation.

\subsection{Notation}

Let $X^{*}=\left(x_{1}^{*}, \ldots, x_{T}^{*}\right)$ be an optimal offline solution that minimizes equation (1) (i.e. the whole workload). Note that $C_{\tau}^{L}\left(X^{*}\right)$ indicates the cost of the optimal solution until $\tau$.

Let $R_{\tau}(X):=\sum_{t=1}^{\tau} f_{t}\left(x_{t}\right)$ with $X=\left(x_{1}, \ldots, x_{\tau}\right)$ denote the operating cost until $\tau$, let $S_{\tau}^{L}(X):=\beta \sum_{t=1}^{\tau}\left(x_{t}-x_{t-1}\right)^{+}$denote the switching cost in $C_{\tau}^{L}(X)$ and let $S_{\tau}^{U}(X):=\beta \sum_{t=1}^{\tau}\left(x_{t-1}-x_{t}\right)^{+}$ denote the switching cost in $C_{\tau}^{U}(X)$. Note that $C_{\tau}^{L}(X)=R_{\tau}(X)+$ $S_{\tau}^{L}(X)$ and $C_{\tau}^{U}(X)=R_{\tau}(X)+S_{\tau}^{U}(X)$. Furthermore,

$$
S_{\tau}^{L}(X)=S_{\tau}^{U}(X)+\beta x_{\tau}
$$

as well as $C_{\tau}^{L}(X)=C_{\tau}^{U}(X)+\beta x_{\tau}$ holds, because in $C_{\tau}^{L}$ we have to pay the missing switching cost to reach the final state $x_{\tau}$. Note that $\beta x_{\tau}$ equals the cost for powering up in $C_{\tau}^{L}$ minus the cost for powering down in $C_{\tau}^{U}$.

Given an arbitrary function $f:[m] \rightarrow \mathbb{R}$, we define

$$
\Delta f(x):=f(x)-f(x-1)
$$

as the slope of $f$ at $x$. Let

$$
\hat{C}_{\tau}^{Y}(x):=\min _{x_{1}, \ldots, x_{\tau-1}} C_{\tau}^{Y}\left(\left(x_{1}, \ldots, x_{\tau-1}, x\right)\right)
$$

with $Y \in\{L, U\}$ be the minimal cost achievable with $x_{\tau}=x$.

\subsection{Competitive ratio}

In this section we prove that the LCP algorithm described by equation (7) achieves a competitive ratio of 3 . First, we show that the optimal solution is bounded by the upper and lower bounds defined in the previous section.

LEMMA 3.1. For all $\tau, x_{\tau}^{L} \leq x_{\tau}^{*} \leq x_{\tau}^{U}$ holds.

Proof. We prove both parts of the inequality by contradiction:

Part $1\left(x_{\tau}^{L} \leq x_{\tau}^{*}\right)$ : Assume that $x_{\tau}^{L}>x_{\tau}^{*}$. By the definition of the lower bound, $C_{\tau}^{L}\left(X_{\tau}^{L}\right)<C_{\tau}^{L}\left(X^{*}\right)$ holds and we can replace $\left(x_{1}^{*}, \ldots, x_{\tau}^{*}\right)$ by $\left(x_{\tau, 1}^{L}, \ldots x_{\tau, \tau}^{L}\right)$. This reduces the total cost of $x^{*}$, because the cost up to $\tau$ is reduced and for $\tau+1$ there are no additional switching costs because $x_{\tau}^{L}>x_{\tau}^{*}$ holds. Thus $x^{*}$ would not be an optimal solution which is a contradiction, so $x_{\tau}^{L} \leq x_{\tau}^{*}$ must be fulfilled.

Part $2\left(x_{\tau}^{*} \leq x_{\tau}^{U}\right)$ : Assume that $x_{\tau}^{*}>x_{\tau}^{U}$. By definition of the upper bound, $C_{\tau}^{U}\left(X_{\tau}^{U}\right)<C_{\tau}^{U}\left(X^{*}\right)$ and, thus,

$$
R_{\tau}\left(X_{\tau}^{U}\right)+S_{\tau}^{U}\left(X^{U}\right)<R_{\tau}\left(X^{*}\right)+S_{\tau}^{U}\left(X^{*}\right)
$$


holds. The cost of the optimal solution until $\tau$ is $R_{\tau}\left(X^{*}\right)+S_{\tau}^{L}\left(X^{*}\right)$. If the states $\left(x_{1}^{*}, \ldots, x_{\tau}^{*}\right)$ are replaced by $X_{\tau}^{U}$ and afterwards $x_{\tau}^{*}-x_{\tau, \tau}^{U}$ servers are powered up (to ensure that we end in the same state), then the cost is $R_{\tau}\left(X_{\tau}^{U}\right)+S_{\tau}^{L}\left(X^{U}\right)+\beta\left(x_{\tau}^{*}-x_{\tau, \tau}^{U}\right)$. This cost must be greater than or equal to the cost of the optimal solution, so

$$
R_{\tau}\left(X_{\tau}^{U}\right)+S_{\tau}^{L}\left(X^{U}\right)+\beta\left(x_{\tau}^{*}-x_{\tau, \tau}^{U}\right) \geq R_{\tau}\left(X^{*}\right)+S_{\tau}^{L}\left(X^{*}\right)
$$

holds. By using equation (8), we get

$$
R_{\tau}\left(X_{\tau}^{U}\right)+S_{\tau}^{U}\left(X^{U}\right)+\beta x_{\tau, \tau}^{U}+\beta\left(x_{\tau}^{*}-x_{\tau, \tau}^{U}\right) \geq R_{\tau}\left(X^{*}\right)+S_{\tau}^{U}\left(X^{*}\right)+\beta x_{\tau}^{*} .
$$

We eliminate identical terms and get

$$
R_{\tau}\left(X_{\tau}^{U}\right)+S_{\tau}^{U}\left(X^{U}\right) \geq R_{\tau}\left(X^{*}\right)+S_{\tau}^{U}\left(X^{*}\right)
$$

which is a contradiction to inequality (9). Therefore our assumption was wrong, so $x_{\tau}^{*} \leq x_{\tau}^{U}$ must be fulfilled.

The following four lemmas show important properties of $\hat{C}_{\tau}^{L}(x)$. First, we prove that the relation between $C_{\tau}^{L}(X)$ and $C_{\tau}^{U}(X)$ described by equation (8) still holds for $\hat{C}_{\tau}^{L}(x)$ and $\hat{C}_{\tau}^{U}(x)$.

Lemma 3.2. For all $\tau, \hat{C}_{\tau}^{L}(x)=\hat{C}_{\tau}^{U}(x)+\beta x$ holds.

Proof. Let $X^{L}$ be a corresponding solution for $\hat{C}_{\tau}^{L}(x)$ such that $C_{\tau}^{L}\left(X^{L}\right)=\hat{C}_{\tau}^{L}(x)$ and let $X^{U}$ be a corresponding solution for $\hat{C}_{\tau}^{U}(x)$ such that $C_{\tau}^{U}\left(X^{U}\right)=\hat{C}_{\tau}^{U}(x)$. Note that the last state of $X^{L}$ and $X^{U}$ is $x$. Since $X^{U}$ is optimal for $C_{\tau}^{U}$, the inequality $C_{\tau}^{U}\left(X^{U}\right) \leq C_{\tau}^{U}(X)$ holds for all $X=\left(x_{1}, \ldots, x_{\tau-1}, x\right)$. By equation (8), we get

$$
C_{\tau}^{L}\left(X^{U}\right)-\beta x \leq C_{\tau}^{L}(X)-\beta x
$$

which is equivalent to $C_{\tau}^{L}\left(X^{U}\right) \leq C_{\tau}^{L}(X)$. With $X:=X^{L}$ we get $C_{\tau}^{L}\left(X^{U}\right) \leq C_{\tau}^{L}\left(X^{L}\right)$. Since $X^{L}$ is optimal for $C_{\tau}^{L}, X^{U}$ must be optimal too, so $C_{\tau}^{L}\left(X^{U}\right)=C_{\tau}^{L}\left(X^{L}\right)$ holds. All in all, we get

$$
\hat{C}_{\tau}^{L}(x)=C_{\tau}^{L}\left(X^{L}\right)=C_{\tau}^{L}\left(X^{U}\right)=C_{\tau}^{U}\left(X^{U}\right)+\beta x=\hat{C}_{\tau}^{U}(x)+\beta x .
$$

Obviously, the cost functions $C_{\tau}^{L}(X)$ and $C_{\tau}^{U}(X)$ are convex, since convexity is closed under addition. The following lemma shows that also $\hat{C}_{\tau}^{L}(x)$ and $\hat{C}_{\tau}^{U}(x)$ are convex.

Lemma 3.3. For all $\tau$ and $Y \in\{L, U\}, \hat{C}_{\tau}^{Y}(x)$ is a convex function.

LEMMA 3.4. The slope of $\hat{C}_{\tau}^{L}(x)$ is at most $\beta$ for $x \leq x_{\tau}^{U}$ and at least $\beta$ for $x>x_{\tau}^{U}$, i.e. $\Delta \hat{C}_{\tau}^{L}\left(x_{\tau}^{U}\right) \leq \beta$ and $\Delta \hat{C}_{\tau}^{L}\left(x_{\tau}^{U}+1\right) \geq \beta$

The proof of Lemma 3.3 and 3.4 can be found in the full paper.

Lemma 3.5. For $x \leq x_{\tau}^{U}$, the slope of $\hat{C}_{\tau}^{L}(x)$ is at most $\beta$, i.e. $\Delta \hat{C}_{\tau}^{L}(x) \leq \beta$ holds.

Proof. By Lemma 3.4 $\Delta \hat{C}_{\tau}^{L}\left(x_{\tau}^{U}\right) \leq \beta$ holds and by Lemma 3.3 $\hat{C}_{\tau}^{L}$ is convex, so $\Delta \hat{C}_{\tau}^{L}(x) \leq \beta$ for $x \leq x_{\tau}^{U}$.

The next lemma characterizes the behavior of the optimal solution backwards in time.

LEMmA 3.6. A solution vector $\left(\hat{x}_{1}, \ldots, \hat{x}_{T}\right)$ that fulfills the following recursive equality for all $t \in\{1, \ldots, T\}$ is optimal:

$$
\hat{x}_{t}:= \begin{cases}0, & t=T+1 \\ {\left[\hat{x}_{t+1}\right]_{x_{t}^{L}}^{x_{t}^{U}},} & t \leq T\end{cases}
$$

Proof. We will prove the lemma by induction in reverse time. Powering down does not cost anything, so setting $\hat{x}_{T+1}=0$, does not produce any additional costs. Assume that $\left(\hat{x}_{\tau+1}, \ldots, \hat{x}_{T}\right)$ can lead to an optimal solution, i.e. there exists an optimal solution $X^{*}$ with $x_{t}^{*}=\hat{x}_{t}$ for $t \geq \tau+1$. We will show that the vector $\left(\hat{x}_{\tau}, \ldots, \hat{x}_{T}\right)$ can still lead to an optimal solution.

We have to examine three cases:

Case 1: If $\hat{x}_{\tau+1}<x_{\tau}^{L}$, then $\hat{x}_{\tau}=x_{\tau}^{L}$. By Lemma 3.1, $x_{\tau}^{*} \geq x_{\tau}^{L}$ holds. Since $X_{\tau}^{L}$ minimizes $C_{\tau}^{L}$, we know that $C_{\tau}^{L}\left(X_{\tau}^{L}\right) \leq C_{\tau}^{L}(X)$ for all $X=\left(x_{1}, \ldots, x_{\tau}\right)$. Thus there is no benefit to use a state $x^{\prime} \geq x_{\tau}^{L}$, because afterwards we have to power down some servers to reach $\hat{x}_{\tau+1}$. Therefore $\hat{x}_{\tau}=x_{\tau}^{L}$ can still lead to an optimal solution.

Case 2: If $\hat{x}_{\tau+1}>x_{\tau}^{U}$, then $\hat{x}_{\tau}=x_{\tau}^{U}$. By Lemma 3.1, $x_{\tau}^{*} \leq x_{\tau}^{U}$ holds. Since $X_{\tau}^{U}$ minimizes $C_{\tau}^{U}$, we know that $C_{\tau}^{U}\left(X_{\tau}^{U}\right) \leq C_{\tau}^{U}(X)$ for all $X$. By using the solution $X_{\tau}^{U}$ and then switching to state $\hat{x}_{\tau+1}$, the resulting cost is

$$
\begin{aligned}
& C_{\tau}^{L}\left(X_{\tau}^{U}\right)+\beta\left(\hat{x}_{\tau+1}-x_{\tau}^{U}\right)+f_{\tau+1}\left(\hat{x}_{\tau+1}\right) \\
= & C_{\tau}^{U}\left(X_{\tau}^{U}\right)+\beta \hat{x}_{\tau+1}+f_{\tau+1}\left(\hat{x}_{\tau+1}\right) \\
\leq & C_{\tau}^{U}(X)+\beta \hat{x}_{\tau+1}+f_{\tau+1}\left(\hat{x}_{\tau+1}\right) \\
= & C_{\tau}^{L}(X)+\beta\left(\hat{x}_{\tau+1}-x_{\tau}\right)+f_{\tau+1}\left(\hat{x}_{\tau+1}\right) .
\end{aligned}
$$

The last line describes the cost until $\tau+1$ by using the vector $X=\left(x_{1}, \ldots, x_{\tau}\right)$ with $x_{\tau} \leq x_{\tau}^{U}$ instead of $X_{\tau}^{U}$. The cost is not reduced by using $X$, so $\hat{x}_{\tau}=x_{\tau}^{U}$ can still lead to an optimal solution.

Case 3: If $x_{\tau}^{L} \leq \hat{x}_{\tau+1} \leq x_{\tau}^{U}$, then $\hat{x}_{\tau}=\hat{x}_{\tau+1}$. Assume that there is a better state $\hat{x}_{\tau}^{-}<\hat{x}_{\tau}$ with $\hat{C}_{\tau}^{L}\left(\hat{x}_{\tau}^{-}\right)<\hat{C}_{\tau}^{L}\left(\hat{x}_{\tau}\right)$. Since $\hat{x}_{\tau+1}$ leads to an optimal solution, after the time slot $\tau$ we have to power up $\hat{x}_{\tau}-\hat{x}_{\tau}^{-}$servers, so even $\hat{C}_{\tau}^{L}\left(\hat{x}_{\tau}^{-}\right)+\beta\left(\hat{x}_{\tau}-\hat{x}_{\tau}^{-}\right)<\hat{C}_{\tau}^{L}\left(\hat{x}_{\tau}\right)$ holds. By Lemma 3.5, we know that the slope of $\hat{C}_{\tau}^{L}(x)$ is at most $\beta$ for $x \leq x_{\tau}^{U}$. This leads to the contradiction $\hat{C}_{\tau}^{L}\left(\hat{x}_{\tau}\right) \leq \hat{C}_{\tau}^{L}\left(\hat{x}_{\tau}^{-}\right)+\beta\left(\hat{x}_{\tau}^{-}-\hat{x}_{\tau}\right)$. Therefore there is no $\hat{x}_{\tau}^{-}$with the desired properties.

The other direction is more simple: Assume that there is a better state $\hat{x}_{\tau}^{+}>\hat{x}_{\tau}$ with $\hat{C}_{\tau}^{L}\left(\hat{x}_{\tau}^{+}\right)<\hat{C}_{\tau}^{L}\left(\hat{x}_{\tau}\right)$, then $x_{\tau}^{L}$ (which minimizes $\hat{C}_{\tau}^{L}$ ) must be greater than $\hat{x}_{\tau}$, because by Lemma $3.3, \hat{C}_{\tau}^{L}$ is a convex function. However, this is a contradiction to $x_{\tau}^{L} \leq \hat{x}_{\tau+1}=\hat{x}_{\tau}$.

In the following $X^{*}=\left(x_{1}^{*}, \ldots, x_{T}^{*}\right)$ denotes an optimal solution that fulfills the recursive equality of Lemma 3.6. The next lemma describes time slots where $X^{\mathrm{LCP}}$ and $X^{*}$ are in same state. Informally, the lemma says that if the LCP curve cuts the optimal solution, then there is one time slot $\tau$ where both solutions are in the same state.

LEMMA 3.7. If $x_{\tau-1}^{L C P}<x_{\tau-1}^{*}$ and $x_{\tau}^{L C P} \geq x_{\tau}^{*}$, then $x_{\tau}^{L C P}=x_{\tau}^{*}$. If $x_{\tau-1}^{L C P}>x_{\tau-1}^{*}$ and $x_{\tau}^{L C P} \leq x_{\tau}^{*}$, then $x_{\tau}^{L C P}=x_{\tau}^{*}$.

Proof. We will only show the first statement of the lemma, since the other one works exactly analogously. Assume that $x_{\tau-1}^{\mathrm{LCP}}<x_{\tau-1}^{*}$ and $x_{\tau}^{\mathrm{LCP}} \geq x_{\tau}^{*}$ holds. We differ between two cases.

Case 1: If $x_{\tau-1}^{\mathrm{LCP}}<x_{\tau}^{\mathrm{LCP}}$, then $x_{\tau}^{\mathrm{LCP}}=x_{\tau}^{L}$ (by the definition of the LCP algorithm). By $x_{\tau}^{\mathrm{LCP}} \geq x_{\tau}^{*}$ and Lemma 3.1 (which says that $x_{\tau}^{L} \leq x_{\tau}^{*}$ ), we get $x_{\tau}^{\mathrm{LCP}}=x_{\tau}^{*}$.

Case 2: If $x_{\tau-1}^{\mathrm{LCP}} \geq x_{\tau}^{\mathrm{LCP}}$, then $x_{\tau-1}^{*}>x_{\tau}^{*}$. By Lemma 3.6, $x_{\tau-1}^{*}=$ $x_{\tau-1}^{L}$ holds which is a contradiction to $x_{\tau-1}^{*}>x_{\tau-1}^{\mathrm{LCP}} \geq x_{\tau-1}^{L}$.

The time slots where $x_{t}^{\mathrm{LCP}}=x_{t}^{*}$ are denoted by $0=t_{0}<t_{1}<$ $\cdots<t_{\kappa}$. Between these time slots it is not possible that $X^{\mathrm{LCP}}$ 
powers one or more servers down and $X^{*}$ powers servers up or vice versa. In the following $[a: b]$ with $a, b \in \mathbb{N}$ denotes the set $\{a, a+1, \ldots, b\}$. Analogously, we define $[a: b[:=\{a, a+1 \ldots, b-1\}$, ]$a: b]:=\{a+1, a+2, \ldots, b\}$ and $] a: b[:=\{a+1, a+2, \ldots b-1\}$.

Lemma 3.8. For all time intervals $] t_{i}: t_{i+1}[$ with $i \geq 0$, either

(i) $x_{\tau}^{L C P}>x_{\tau}^{*}$ and both $x_{\tau}^{L C P}$ and $x_{\tau}^{*}$ are non-increasing for all $\tau \in] t_{i}: t_{i+1}[$, or

(ii) $x_{\tau}^{L C P}<x_{\tau}^{*}$ and both $x_{\tau}^{L C P}$ and $x_{\tau}^{*}$ are non-decreasing for all $\tau \in] t_{i}: t_{i+1}[$.

Proof. First, we consider the case (i), i.e. $x_{\tau}^{\mathrm{LCP}}>x_{\tau}^{*}$.

If $x_{\tau+1}^{\mathrm{LCP}}>x_{\tau}^{\mathrm{LCP}}$, then $x_{\tau+1}^{L}=x_{\tau+1}^{\mathrm{LCP}}$ by the LCP algorithm and $x_{\tau+1}^{*} \geq x_{\tau+1}^{L}$ by Lemma 3.1. By Lemma 3.6, we get $x_{\tau}^{U}=x_{\tau}^{*}$ which leads to the contradiction $x_{\tau}^{U}=x_{\tau}^{*}<x_{\tau}^{\mathrm{LCP}} \leq x_{\tau}^{U}$ (the last inequality uses the definition of the LCP algorithm). Thus $x_{\tau}^{\mathrm{LCP}}$ is non-increasing for all $\tau \in] t_{i}: t_{i+1}[$.

If $x_{\tau+1}^{*}>x_{\tau}^{*}$, then $x_{\tau}^{*}=x_{\tau}^{U}$ by Lemma 3.6 which is a contradiction to $x_{\tau}^{U} \geq x_{\tau}^{\mathrm{LCP}}>x_{t}^{*}$, so $x_{\tau}^{*}$ is also non-increasing for all $\tau \in] t_{i}: t_{i+1}\left[\right.$. By Lemma 3.7 the inequality $x_{\tau}^{\mathrm{LCP}}>x_{\tau}^{*}$ is fulfilled for all $\tau \in] t_{i}: t_{i+1}[$.

Case (ii) works analogously.

Now we can calculate the switching cost of the LCP algorithm.

Lemma 3.9. $S_{T}^{L}\left(X^{L C P}\right) \leq S_{T}^{L}\left(X^{*}\right)$

Proof. By Lemma 3.8, both $x_{\tau}^{\mathrm{LCP}}$ and $x_{\tau}^{*}$ are either non-increasing or non-decreasing until there is a time slot $t$ with $x_{t}^{\mathrm{LCP}}=x_{t}^{*}$. Therefore, the switching cost during the time interval $\left[t_{i}: t_{i+1}\right]$ is $\beta\left(x_{t_{i}}^{*}-x_{t_{i-1}}^{*}\right)^{+}$for both $X^{\mathrm{LCP}}$ and $X^{*}$. At the end of the workload, $X^{\mathrm{LCP}}$ and $X^{*}$ are maybe in different states. If $x_{T}^{\mathrm{LCP}}<x_{T}^{*}$, then $x_{\tau}^{\mathrm{LCP}}$ and $x_{\tau}^{*}$ are non-decreasing in the corresponding time interval $\left[t_{\kappa}: T\right]$, so the switching cost of LCP is less than the switching cost of the optimal solution. If the $x_{T}^{\mathrm{LCP}}>x_{T}^{*}$, both $x_{\tau}^{\mathrm{LCP}}$ and $x_{\tau}^{*}$ are non-increasing, so there are no switching costs for this time interval. All in all, we get $S_{T}^{L}\left(X^{\mathrm{LCP}}\right) \leq S_{T}^{L}\left(X^{*}\right)$.

Lemma 3.8 divides the intervals $\left[t_{i}: t_{i+1}\right.$ [ into two sets: Intervals of case (i) are called decreasing intervals, the set of those intervals is denoted by $\mathcal{T}^{-}$. Intervals of case (ii) are called increasing intervals and the set is denoted by $\mathcal{T}^{+}$. The following lemma is needed to estimate the operating cost of the LCP algorithm.

Lemma 3.10. For all $\tau \in\left[t_{i}: t_{i+1}\left[\in \mathcal{T}^{+}\right.\right.$,

$$
\hat{C}_{\tau}^{L}\left(x_{\tau}^{L C P}\right)+f_{\tau+1}\left(x_{\tau+1}^{L C P}\right) \leq \hat{C}_{\tau+1}^{L}\left(x_{\tau+1}^{L C P}\right) .
$$

Analogously, for all $\tau \in\left[t_{i}: t_{i+1}\left[\in \mathcal{T}^{-}\right.\right.$,

$$
\hat{C}_{\tau}^{U}\left(x_{\tau}^{L C P}\right)+f_{\tau+1}\left(x_{\tau+1}^{L C P}\right) \leq \hat{C}_{\tau+1}^{U}\left(x_{\tau+1}^{L C P}\right) .
$$

The lemma can be proven by using the properties of $\hat{C}_{\tau}^{L}$ and $\hat{C}_{\tau}^{U}$ shown in Lemma 3.3 and 3.5, the detailed proof can be found in the full version of this paper. We can use Lemma 3.10 to estimate the operating cost of the LCP algorithm.

$$
\text { LEMmA 3.11. } R_{T}\left(X^{L C P}\right) \leq R_{T}\left(X^{*}\right)+\beta \sum_{t=1}^{T}\left|x_{t}^{*}-x_{t-1}^{*}\right|
$$

Proof. Consider the time interval $\left[t_{i}: t_{i+1}\left[\in \mathcal{T}^{+}\right.\right.$. By adding the inequalities of Lemma 3.10 for $\tau \in\left[t_{i}: t_{i+1}\right.$ [, we get

$$
\sum_{t=t_{i}}^{t_{i+1}-1} \hat{C}_{t}^{L}\left(x_{t}^{\mathrm{LCP}}\right)+\sum_{t=t_{i}}^{t_{i+1}-1} f_{t+1}\left(x_{t+1}^{\mathrm{LCP}}\right) \leq \sum_{t=t_{i}}^{t_{i+1}-1} \hat{C}_{t+1}^{L}\left(x_{t+1}^{\mathrm{LCP}}\right)
$$

Subtracting the first sum gives

$$
\begin{gathered}
\sum_{t=t_{i}}^{t_{i+1}-1} f_{t+1}\left(x_{t+1}^{\mathrm{LCP}}\right) \leq \hat{C}_{t_{i+1}}^{L}\left(x_{t_{i+1}}^{\mathrm{LCP}}\right)-\hat{C}_{t_{i}}^{L}\left(x_{t_{i}}^{\mathrm{LCP}}\right) \\
=\hat{C}_{t_{i+1}}^{L}\left(x_{t_{i+1}}^{*}\right)-\hat{C}_{t_{i}}^{L}\left(x_{t_{i}}^{*}\right)=\sum_{t=t_{i}}^{t_{i+1}-1} f_{t+1}\left(x_{t+1}^{*}\right)+\beta\left(x_{t_{i+1}}^{*}-x_{t_{i}}^{*}\right)
\end{gathered}
$$

The first equality holds because $x_{t_{i}}^{\mathrm{LCP}}=x_{t_{i}}^{*}$ and $x_{t_{i+1}}^{\mathrm{LCP}}=x_{t_{i+1}}^{*}$. Considering the time interval $\left[t_{i}: t_{i+1}\left[\in \mathcal{T}^{-}\right.\right.$yields to the following inequality:

$$
\begin{gathered}
\sum_{t=t_{i}}^{t_{i+1}-1} f_{t+1}\left(x_{t+1}^{\mathrm{LCP}}\right) \leq \hat{C}_{t_{i+1}}^{U}\left(x_{t_{i+1}}^{\mathrm{LCP}}\right)-\hat{C}_{t_{i}}^{U}\left(x_{t_{i}}^{\mathrm{LCP}}\right) \\
=\hat{C}_{t_{i+1}}^{U}\left(x_{t_{i+1}}^{*}\right)-\hat{C}_{t_{i}}^{U}\left(x_{t_{i}}^{*}\right)=\sum_{t=t_{i}}^{t_{i+1}-1} f_{t+1}\left(x_{t+1}^{*}\right)+\beta\left(x_{t_{i}}^{*}-x_{t_{i+1}}^{*}\right)
\end{gathered}
$$

In both (12) and (13) the factor after $\beta$ is positive, so we can write

$$
\begin{aligned}
\sum_{t=t_{i}}^{t_{i+1}-1} f_{t+1}\left(x_{t+1}^{\mathrm{LCP}}\right) & \leq \sum_{t=t_{i}}^{t_{i+1}-1} f_{t+1}\left(x_{t+1}^{*}\right)+\beta\left|x_{t_{i+1}}^{*}-x_{t_{i}}^{*}\right| \\
& =\sum_{t=t_{i}}^{t_{i+1}-1} f_{t+1}\left(x_{t+1}^{*}\right)+\beta \sum_{t=t_{i}}^{t_{i+1}-1}\left|x_{t+1}^{*}-x_{t}^{*}\right|
\end{aligned}
$$

By adding all intervals in $\mathcal{T}^{+} \cup \mathcal{T}^{-}$we get

$$
\sum_{t=1}^{T} f_{t}\left(x_{t}^{\mathrm{LCP}}\right) \leq \sum_{t=1}^{T} f_{t}\left(x_{t}^{*}\right)+\beta \sum_{t=1}^{T}\left|x_{t}^{*}-x_{t-1}^{*}\right|
$$

The term $\beta \sum_{t=1}^{T}\left|x_{t}^{*}-x_{t-1}^{*}\right|$ in Lemma 3.11 is upper bounded by twice the switching cost of the optimal schedule:

LEMMA 3.12. $\beta \sum_{t=1}^{T}\left|x_{t}^{*}-x_{t-1}^{*}\right| \leq 2 \cdot S_{T}^{L}\left(X^{*}\right)$

This lemma can be proven with simple calculations (see full paper). Now, we are able to show that LCP is 3-competitive.

Theorem 3.13. The LCP algorithm is 3-competitive.

Proof. By using Lemma 3.9, 3.11 and 3.12 we get

$$
\begin{aligned}
C_{T}^{L}\left(X^{\mathrm{LCP}}\right) & =R_{T}\left(X^{\mathrm{LCP}}\right)+S_{T}^{L}\left(X^{\mathrm{LCP}}\right) \\
& \leq R_{T}\left(X^{*}\right)+\beta \sum_{t=1}^{T}\left|x_{t}^{*}-x_{t-1}^{*}\right|+S_{T}^{L}\left(X^{*}\right) \\
& \leq R_{T}\left(X^{*}\right)+3 \cdot S_{T}^{L}\left(X^{*}\right) \leq C_{T}^{L}\left(X^{*}\right) .
\end{aligned}
$$

\section{LOWER BOUNDS}

In this section we will show lower bounds for both the discrete and continuous data-center optimization problem. First, we prove that there is no deterministic online algorithm that achieves a competitive ratio better than 3 for the discrete problem. This lower bound demonstrates that the LCP algorithm analyzed in the previous section is optimal. Afterwards, we show that this lower bound 
also holds for Lin's model (introduced in [17]) that is a bit more restricted than the general model investigated in the previous sections. A formal definition of Lin's model is given in Section 4.2. Moreover, we give a lower bound for the continuous setting and show that this lower bound holds again for Lin's model. A lower bound of 2 for the general continuous setting was independently shown by Antoniadis et. al. [3]. Finally, we extend our lower bounds to the scenario that an online algorithm has a finite prediction window.

To simplify the analysis, the switching costs are paid for both powering up and powering down. At the end of the workload all servers have to be powered down. This ensures that the total cost remains the same. We will set $\beta=2$, so changing a server's state will cost $\beta / 2=1$. Thus the cost of a schedule is defined by

$$
C(X):=\sum_{t=1}^{T} f_{t}\left(x_{t}\right)+\sum_{t=1}^{T+1}\left|x_{t}-x_{t-1}\right|
$$

with $x_{0}:=x_{T+1}:=0$.

\subsection{Discrete setting}

First, we analyze the discrete setting.

THEOREM 4.1. There is no deterministic online algorithm that achieves a competitive ratio of $c<3$ for the discrete data-center optimization problem.

Proof. Assume that there is a deterministic algorithm $\mathcal{A}$ that is $(3-\delta)$-competitive with $\delta>0$. The adversary will use the functions $\varphi_{0}(x)=\epsilon|x|$ and $\varphi_{1}(x)=\epsilon|x-1|$ with $\epsilon \rightarrow 0$, so we only need the states 0 and 1 , there is no benefit to use other states. If $\mathcal{A}$ is in state 0 or 1 , the adversary will send $\varphi_{1}$ or $\varphi_{0}$, respectively.

Let $S$ be the number of time slots where algorithm $\mathcal{A}$ changes the state of a server, i.e. $S$ is the switching cost of $\mathcal{A}$. Let $T$ be length of the whole workload (we will define $T$ later), so for $T-S$ time slots the operating costs of $\mathcal{A}$ are $\epsilon$. Thus, the total cost of $\mathcal{A}$ is

$$
C(\mathcal{A})=(T-S) \epsilon+S .
$$

The cost of the optimal offline schedule can be bounded by the minimum of the following two strategies. The first strategy is to stay at one state for the whole workload. If $\varphi_{0}$ is sent more often than $\varphi_{1}$, then this is state 0 , else it is state 1 . The operating cost is at most $T \epsilon / 2$, the switching cost is at most 2, because if we use state 1 , we have to switch the state at the beginning and end of the workload. The second strategy is to always switch the state, such that there are no operating costs. In this case the switching cost is at most $S+2$, because we switch the state after each time $\mathcal{A}$ switches its state as well as possibly at the beginning and the end of the workload. Thus, the cost of the optimal offline schedule is

$$
C\left(X^{*}\right) \leq \min (T \epsilon / 2+2, S+2) \text {. }
$$

We want to find a lower bound for the competitive ratio $\frac{C(\mathcal{A})}{C\left(X^{*}\right)}$. We distinguish between $S \geq T \epsilon / 2$ (case 1 ) and $S<T \epsilon / 2$ (case 2).

In case 1 the competitive ratio of $\mathcal{A}$ is

$$
\begin{aligned}
\frac{C(\mathcal{A})}{C\left(X^{*}\right)} \stackrel{(14)}{\geq} \frac{(T-S) \epsilon+S}{T \epsilon / 2+2}=2+\frac{S(1-\epsilon)-4}{T \epsilon / 2+2} \\
\\
\geq 2+\frac{(T \epsilon / 2)(1-\epsilon)-4}{T \epsilon / 2+2}=2+(1-\epsilon)-\frac{2(1-\epsilon)+4}{T \epsilon / 2+2}
\end{aligned}
$$

The last inequality uses $S \geq T \epsilon / 2$ that holds for case 1 . By setting $T \geq \frac{1}{\epsilon^{2}}$, we get $\lim _{\epsilon \rightarrow 0} T \epsilon=\infty$ and thus $\lim _{\epsilon \rightarrow 0} \frac{C(\mathcal{A})}{C\left(X^{*}\right)}=3$.

In case 2 , we get

$$
\begin{aligned}
\frac{C(\mathcal{A})}{C\left(X^{*}\right)} & \stackrel{(14)}{\geq} \frac{(T-S) \epsilon+S}{S+2}=(1-\epsilon)+\frac{T \epsilon-2(1-\epsilon)}{S+2} \\
& \geq(1-\epsilon)+\frac{T \epsilon-2(1-\epsilon)}{T \epsilon / 2+2}=3-\epsilon-\frac{2(1-\epsilon)+4}{T \epsilon / 2+2}
\end{aligned}
$$

Again, we set $T \geq \frac{1}{\epsilon^{2}}$ and get $\lim _{\epsilon \rightarrow 0} \frac{C(\mathcal{A})}{C\left(X^{*}\right)}=3$.

Therefore there is no algorithm with a competitive ratio that is less than 3 . We can set $T$ to an arbitrarily large value, so the total cost of $\mathcal{A}$ converges to infinity.

\subsection{Discrete setting: Lin's model}

Lin et. al. [17] introduced a more restricted setting as described by equation (2). In this section we show that the lower bound of 3 still holds for this model. The essential differences of Lin's model to the general model are: (1) There is only one convex function for the whole problem instance and (2) there is the additional condition that $x_{t} \geq \lambda_{t}$. The different definition of the switching cost does not influence the total cost as already mentioned in the beginning of Section 4 .

THeOREM 4.2. There is no deterministic online algorithm for the discrete setting of Lin's model with a competitive ratio of $c<3$.

Proof. The general model (examined in the previous sections) is denoted by $\mathcal{G}$ and Lin's model is denoted by $\mathcal{L}$. The states of the model $\mathcal{X} \in\{\mathcal{G}, \mathcal{L}\}$ are indicated by $x_{t}^{X}$. We will use the same idea as in the proof of Theorem 4.1, but we have to modify it such that it fits for Lin's model.

We use 2 servers, so the states are $x_{t}^{\mathcal{L}} \in\{0,1,2\}$. Instead of switching between the states 0 and 1 in $\mathcal{G}$, we will switch between 1 and 2 in $\mathcal{L}$, so for $t \in\{1, \ldots, T\}$ we have $x_{t}^{\mathcal{L}}=x_{t}^{\mathcal{G}}+1$. In $\mathcal{L}$ the state 0 is only used at the beginning $(t=0)$ of the workload. This leads to additional switching costs of 1 for both the optimal offline solution and the online algorithm. However, for a sufficiently long workload the total cost converges to infinity, so the constant extra cost does not influence the competitive ratio.

We will apply the same adversary strategy used in the proof of Theorem 4.1. Let $f(z):=\epsilon|1-2 z|$ with $\epsilon \rightarrow 0$, let $\beta=2$. If the adversary in $\mathcal{G}$ sends $\varphi_{0}(x)=\epsilon|x|$ as function, then we will use $\lambda_{t}=l_{0}:=0.5$ which leads to operating cost of

$$
x_{t}^{\mathcal{L}} f\left(l_{0} / x_{t}^{\mathcal{L}}\right)=x_{t}^{\mathcal{L}} \cdot \epsilon\left|1-\frac{1}{x_{t}^{\mathcal{L}}}\right|=\epsilon\left|x_{t}^{\mathcal{L}}-1\right|=\epsilon\left|x_{t}^{\mathcal{G}}\right|
$$

If the adversary sends $\varphi_{1}(x)=\epsilon|1-x|$, then we will use $\lambda_{t}=l_{1}=1$ which leads to operating cost of

$$
x_{t}^{\mathcal{L}} f\left(l_{1} / x_{t}^{\mathcal{L}}\right)=x_{t}^{\mathcal{L}} \cdot \epsilon\left|1-\frac{2}{x_{t}^{\mathcal{L}}}\right|=\epsilon\left|x_{t}^{\mathcal{L}}-2\right|=\epsilon\left|1-x_{t}^{\mathcal{G}}\right|
$$

Thus the difference (1) between both models is solved.

For $t \geq 1$ it is not allowed to use the state $x_{t}^{\mathcal{L}}=0$, because both $l_{0}$ and $l_{1}$ are greater than 0 . For $x_{t}^{\mathcal{L}} \in\{1,2\}$ the inequality $x_{t} \geq \lambda_{t}$ is always fulfilled, so the difference (2) is solved too. 


\subsection{Continuous setting}

In this section we analyze the continuous setting of the data-center optimization problem.

Theorem 4.3. There is no deterministic online algorithm for the continuous data-center optimization problem that achieves a competitive ratio that is less than 2 .

The proof consists of two parts. First we will construct an algorithm $\mathcal{B}$ whose competitive ratio is greater than $2-\delta$ for an arbitrary small $\delta$. Then we will show that the competitive ratio of any deterministic algorithm that differs from $\mathcal{B}$ is greater than 2 .

For the first part we use an adversary that uses $\varphi_{0}(x)=\epsilon|x|$ and $\varphi_{1}(x)=\epsilon|1-x|$ as functions where $\epsilon \rightarrow 0$. Let $b_{t}$ be the state of $\mathcal{B}$ at time $t$. If the function $\varphi_{0}$ arrives, then the next state $b_{t+1}$ is $\max \left\{b_{t}-\epsilon / 2,0\right\}$. If $\varphi_{1}$ arrives, the next state is $b_{t+1}:=$ $\min \left\{b_{t}+\epsilon / 2,1\right\}$. The algorithm starts at $b_{0}=0$, so $b_{t} \in[0,1]$ is fulfilled for all $t$. Note that algorithm $\mathcal{B}$ is equivalent to Bansal's algorithm given in [5] for the special case of $\varphi_{0}$ and $\varphi_{1}$ functions. As shown in the full version of this paper, the competitive ratio of $\mathcal{B}$ is at least 2 .

For the second part we use the following adversary strategy. Let $a_{t}$ be the state of $\mathcal{A}$ at time $t$. If $a_{t}<b_{t}$ or $a_{t}=0$, the adversary sends $\varphi_{1}$ as next function. If $a_{t}>b_{t}$ or $a_{t}=1$, the adversary sends $\varphi_{0}$. For the other cases the adversary can choose an arbitrary function. By doing this, it can be shown that the cost of $\mathcal{A}$ is always greater than or equal to the cost of $\mathcal{B}$, so the competitive ratio of $\mathcal{A}$ is at least 2. The detailed analysis can be found in the full paper.

\subsection{Continuous setting: Lin's model}

Analogously to the discrete setting, in this section we want to show that the lower bound of 2 for the continuous data-center optimization problem still holds for Lin's model described in Section 4.3.

THEOREM 4.4. There is no deterministic online algorithm for the continuous setting of Lin's model with a competitive ratio of $c<2$.

To prove this theorem we will apply the same adversary strategy used in the previous section. We set $f(z):=\epsilon|1-k z|$ with $\epsilon \rightarrow 0$ and $k \rightarrow \infty$. If the adversary in the general model sends $\varphi_{0}(x)=\epsilon|x|$ as function, then we will use $\lambda_{t}=0$, if it sends $\varphi_{1}(x)=\epsilon|1-x|$, then we will use $\lambda_{t}=1 / k$. As shown in the full paper, this leads to the same operating cost functions as in the general model.

\subsection{Online algorithms with prediction window}

So far, we have considered online algorithms that at time $t$ only know the arriving function $f_{t}$ in determining the next state. In contrast, an offline algorithm knows the whole function sequence $F$. There are models between these edge cases. An online algorithm with a prediction window of length $w$, at any time $t$, can not only use the function $f_{t}$ but the function set $\left\{f_{t}, \ldots, f_{t+w}\right\}$ to choose the state $x_{t}$. This problem extension was also defined by Lin et al. $[17,19]$. If $w$ has a constant size (i.e. $w$ is independent of $T$ ), then the lower bounds developed in the previous sections still holds as the following theorem shows. We develop the lower bounds for Lin's model so that they hold for the general model as well.

Theorem 4.5. Let $w \in \mathbb{N}$ and $\delta>0$ be arbitrary constants. There is no deterministic online algorithm with a prediction window of length $w$ that achieves a competitive ratio of $3-\delta$ in the discrete setting or $2-\delta$ in the continuous setting for Lin's model.

The basic idea for proving this theorem is to use a worst case workload for online algorithms without prediction window and send a scaled version of each function several times (dependent on $w$ ), such that there is no advantage in knowing the next $w$ functions. Details can be found in the full version of this paper.

\section{REFERENCES}

[1] L.L.H. Andrew, S. Barman, K. Ligett, M. Lin, A. Meyerson, A. Roytman and A. Wierman. A tale of two metrics: Simultaneous bounds on competitiveness and regret. Proc. 26th Annual Conference on Learning Theory (COLT'13), 741-763, 2013.

[2] A. Antoniadis, N. Barcelo, M. Nugent, K. Pruhs, K. Schewior and M. Scquizzato. Chasing convex bodies and functions. Proc. 12th Latin American Symposium on Theoretical Informatics (LATIN'16), 68-81, 2016.

[3] A. Antoniadis and K. Schewior. A tight lower bound for online convex optimization with switching costs. Announced at the 15th Workshop on Approximation and Online Algorithms (WAOA'17), 2017.

[4] M. Armbrust, A. Fox, R. Griffith, A.D. Joseph, R.H. Katz, A. Konwinski, G. Lee, D.A. Patterson, A. Rabkin, I. Stoica and M. Zaharia. Above the clouds: A Berkeley view of cloud computing. Technical Report No. UCB/EECS-2009-28. EECS Department, University of California, Berkeley, 2009.

[5] N. Bansal, A. Gupta, R. Krishnaswamy, K. Pruhs, K. Schewior and C. Stein. A 2-competitive algorithm for online convex optimization with switching costs. Proc. 18th. International Workshop on Approximation Algorithms for Combinatorial Optimization Problems (APPROX15), 96-109, 2015.

[6] L.A. Barroso and U. Hölzle. The case for energy-proportional computing. IEEE Computer, 40(12):33-37, 2007

[7] T. Bawden. Global warming: Data centres to consume three times as much energy in next decade, experts warn. http: //www. independent. co.uk/environment/global-warming-datacentres-to-consume-three-times-as-much-energy-in-next-decadeexperts-warn-a6830086. html, 2016.

[8] K.G. Brill. The Invisible Crisis in the Data Center: The Economic Meltdown of Moore's Law. white paper, Uptime Institute, 2-5, 2007.

[9] M. Dayarathna, Y. Wen and R. Fan. Data center energy consumption modeling: A survey. IEEE Communications Surveys and Tutorials, 18(1):732-794, 2016.

[10] A. Gandhi and M. Harchol-Balter. How data center size impacts the effectiveness of dynamic power management. 49th Annual Allerton Conference, 1164-1169, 2011.

[11] A. Gandhi, M. Harchol-Balter and I.J.B.F. Adan. Server farms with setup costs. Perform. Eval., 67(11):1123-1138, 2010.

[12] Z.J. Haas and S. Gu. On power management policies for data centers. Proc. IEEE Int. Conf. on Data Science and Data Intensive Systems (DSDIS), 404-411, 2015.

[13] J. Hamilton. Cost of Power in Large-Scale Data Centers. http://perspectives. mvdirona. com/2008/11/cost-of-power-in-largescale-data-centers/

[14] S. Khuller, J. Li and B. Saha. Energy efficient scheduling via partial shutdown. Proc. 21st Annual ACM-SIAM Symposium on Discrete Algorithms, 1360-1372, 2010.

[15] J. Li and S. Khuller. Generalized machine activation problems. Proc. 22nd Annual ACM-SIAM Symposium on Discrete Algorithms, 80-94, 2011.

[16] M. Lin, Z. Liu, A. Wierman and L.L.H. Andrew. Online algorithms for geographical load balancing. Proc. International Green Computing Conference (IGCC'12), 1-10, 2012.

[17] M. Lin, A. Wierman, L.L.H. Andrew and E. Thereska. Dynamic right-sizing for power-proportional data centers. Proc. 30th IEEE International Conference on Computer Communications (INFOCOM'11), 1098-1106, 2011.

[18] M. Lin, A. Wierman, L.L.H. Andrew and E. Thereska. Online dynamic capacity provisioning in data centers. Proc. 49th Annual Allerton Conference on Communication, Control, and Computing, 1159-1163, 2011.

[19] M. Lin, A. Wierman, L.L.H. Andrew and E. Thereska. Dynamic right-sizing for power-proportional data centers. IEEE/ACM Trans. Netw.,21(5):1378-1391, 2013.

[20] Z. Liu, M. Lin, A. Wierman, S.H. Low, L.L.H. Andrew. Greening geographical load balancing. IEEE/ACM Trans. Netw., 23(2):657-671, 2015.

[21] P. Schmid and A. Roos. Overclocking Core i7: Power Versus Performance. Idle/Peak Power Consumption Analysis. http://www. tomshardware.com/reviews/overclock-core-i 7, 2268-10. html, 2009.

[22] A. Shehabi, S. Smith, D. Sartor, R. Brown, M. Herrlin, J. Koomey, E. Masanet, N. Horner, I. Azevedo and W. Lintner. United States data center energy usage report. Lawrence Berkeley National Laboratory, LBNL-1005775, 2016.

[23] K. Wang, M. Lin, F. Ciucu, A. Wierman and C. Lin. Characterizing the impact of the workload on the value of dynamic resizing in data centers. Perform. Eval., $85-86: 1-18,2015$ 